\title{
Keep On Fighting: The Dynamics of Head Starts in All-Pay Auctions*
}

\author{
Derek J. Clark and Tore Nilssen ${ }^{\dagger}$
}

May 3, 2018

\begin{abstract}
We investigate a model of a series of contests in which a contestant's past and present success gives a head start over a rival in the future. How this advantage from winning affects contestants' efforts, whether the laggard gives up or keeps on fighting, and how the head start develops over time, are key issues. We find that the expected effort of the laggard will always be higher than the rival at some stage in the series of contests, and this is most likely to happen when at a large disadvantage or at a late stage in the series.

Keywords: contest; all-pay auction; win advantage; head start.

JEL codes: D74, D72
\end{abstract}

${ }^{*}$ We are grateful for comments received from an advisory editor and three anonymous referees, as well as from Rolf Aaberge, Dan Kovenock, and audiences at the 2015 Royal Economic Society Conference in Manchester, the conference on "Contests: Theory and Evidence" at the University of East Anglia, the 2015 Econometric Society World Congress in Montreal, and a seminar at the University of Oslo. We are especially indebted to Jan Yngve Sand for many good discussions on the topic of this paper. Nilssen's research has received funding from the ESOP Centre at the University of Oslo, which was supported by the Research Council of Norway through its Centres of Excellence funding scheme, project number 179552 .

${ }^{\dagger}$ Clark: School of Business and Economics, University of Troms $\varnothing$, NO-9037 Troms $\varnothing$, Norway; derek.clark@uit.no. Nilssen: Department of Economics, University of Oslo, P.O. Box 1095 Blindern, NO-0317 Oslo, Norway; tore.nilssen@econ.uio.no. 


\section{Introduction}

Winning a competition may result not only in a prize, but also in an advantage in subsequent competitions. Consider, for example, competitions for research grants. While the successful applicant for a grant may harvest all the direct benefits that the research money awarded provides, there may also be an extra benefit from winning: carrying out the research that the original grant facilitates makes for increased chances to win in future grant competitions. In this way, an early competition for a prize implies that there will be advantaged and disadvantaged participants in subsequent competitions: winning an early contest gives you a head start in later contests. Questions are then how contestants' incentives to put in effort in such sequential competitions vary over time as successes and failures are recorded, and how these incentives interact in the shaping and development of head starts.

In order to understand the dynamics of this kind of competition, we develop in this paper a two-player model of a series of contests where, in each stage contest, there is a prize to win of common value. A player wins a stage contest by having a larger score than the opponent, where a player's score consists of effort in the current contest and the number of contests that the player has won so far; we modify the number of wins by a parameter that captures the importance of winning, and this is assumed identical for the players. ${ }^{1}$ Hence, the player with larger number of wins has a head start in any stage contest since he can win now even when investing less effort than the rival. We point out two forces that interact in explaining contestants' incentives across time. On one hand, there is a large incentive to be the player with the head start since this can save effort costs in the future without necessarily reducing the probability of success. However, the head start creates an asymmetry which lowers both players' incentives to put in effort, but more so for the disadvantaged player - the laggard. This is because the head start enables the advantaged player - the leader - to lay back a bit and still stand a good chance to win again, so that also the laggard pulls back somewhat.

On the other hand, there is an additional value of being the leader. A new win means he will also be a leader in the future, while a win for the laggard will at best even the score. The difference in value between winning and losing at each stage is therefore larger for the leader than for the laggard. A win for the laggard will at best even the score (with an expected payoff of zero), and a loss means that the laggard is still at a disadvantage (with the same expected payoff of zero). This dampens the laggard's incentives to put in effort. However, the value of winning

\footnotetext{
${ }^{1}$ Clark and Nilssen (2018) consider a series of two all-pay auctions in which the value of winning the first contest can give the players different head starts in the second.
} 
and continuing as leader falls over time in a game of finite length, simply because there are fewer future contests left; hence, even the leader will put in less effort at some stage in the contest sequence. This encourages the laggard who, towards the end of the sequence of contests, will be the high performer. The balance of these effects - and the interplay between them - cannot be captured in a one-shot game with a head start.

Above, we mentioned the advantage that winning a current research grant can convey on the winner in future competitions. It is the actual fact of winning that creates the advantage here; whether an application is a little better or much better than that of the opponent is not relevant. Winning an early grant, and being able to carry out a research program, enhances the chance to win again in the competition for later grants. Winning gives a player a head start in the next contest. This can also be expected to occur in a number of other contexts. In sales-force management, it is customary to give awards to the Seller of the Month and the like. And in such sales forces, it is not uncommon for the more successful agents to be given less administrative duties, better access to back-office resources, more training than the less successful, and better territories; see, e.g., Skiera and Albers (1998), Farrell and Hakstian (2001), and Krishnamoorthy, et al. (2005). Being a winning salesperson gives a head start in later contests.

In our model, winning creates a head start advantage. Another strand of the literature considers that winning agents have access to different prizes than less successful ones (Megidish and Sela, 2014). Also a psychological momentum may accrue to winners (Krumer, 2013). Experiments carried out by Eriksson, et al. (2009) indicate that laggards keep on fighting. In particular, the authors compare laggards who get feedback, i.e., are informed that they are lagging, with ones who do not get such feedback and find that informed laggards are more prone to put in effort. This finding fits well with our theoretical predictions if we view winning a competition in these experiments as getting a momentum before later competitions.

The sequence of contests that we model in this paper gives, as noted, rise to the creation of a leader and a laggard by which the winner of an early contest gets an advantage in the next one. Such dynamic win effects, in various forms, are also discussed by Krumer (2013), Megidish and Sela (2014), and Clark, et al. (2018). Krumer's (2013) discussion is in the context of a race, whereas the other two papers are on sequences of Tullock contests. These contributions are confined to analyzing sequences of two stage competitions. In the present study, on the other hand, we allow for longer sequences, and the stage contest is an all-pay auction. With long sequences of contests, we are able to discuss how the interaction of the leader and the laggard's incentives develop over time.

Leaders and laggards also feature in races, i.e., best-of- $t$ contests, where the overall winner is the first to win $t$ stage contests; see Harris and Vickers 
(1987) for an early analysis and Konrad (2009) for an overview. ${ }^{2}$ The winner of the first stage of a race becomes the leader in the second, in the sense of having fewer stages left to complete the game. This leader has a much firmer grip on the rest of the game than the leader has in our context. Results differ in the two set-ups, not surprisingly. While the laggard is strongly discouraged in a race, he is much more interested in staying and keep on fighting in our setting.

Strumpf (2002), Konrad and Kovenock (2009, 2010), Fu, et al. (2015), and Konrad (2018) show various ways in which the discouragement of the laggard can be mitigated in a race. In Strumpf (2002), this happens when the contests most valuable to the laggard are late in the race; in Konrad and Kovenock (2009), it happens because of the introduction of stage prizes; in Konrad and Kovenock (2010) because of the introduction of uncertainty; in $\mathrm{Fu}$, et al. (2015) because of the introduction of team competition; and in Konrad (2018) because of players having fixed budgets over the sequence of contests. In these papers, there is no dynamic win effect. While the discouragement of the laggard is mitigated, he never exerts the higher expected effort, as he eventually does in our analysis. Of these papers, Konrad and Kovenock (2009) is particularly closely related to the present work, and we therefore include a more detailed discussion of this relationship in Section 6 below.

Gelder (2014), on the other hand, shows, in his analysis of a race, that a combination of punishment from loss and discounting creates a scope for what he calls "last stand" behavior. This resembles our result that the laggard exerts the higher expected effort in the final period, and often also earlier. Gelder thus establishes an equilibrium outcome similar to ours, but in a model of a race where the laggard's position, and therefore also his incentives, are different from our model. In addition, we get this outcome even when there is no discounting.

Bergerhoff and Vosen (2015) introduce reference-dependent preferences and find this to create what they call turn-around equilibria, where the disadvantaged player has the higher probability of winning. ${ }^{3}$ In our analysis, preferences are standard, and while the laggard exerts the higher expected effort towards the end of the game, his probability of winning is always lower than that of the leader.

A phenomenon related to the dynamic win effect that we consider here are dynamic effort effects, where efforts in an early contest, rather than

\footnotetext{
${ }^{2}$ Another interesting multi-period situation creating a leader and a laggard is the incumbency competition, where the winner of contest $t$ becomes the leader at contest $t+1$; see, e.g., Ofek and Sarvary (2003) and Mehlum and Moene (2006, 2016).

${ }^{3}$ This study relates to Berger and Pope's (2011) study of winning laggards in professional baseball, and to Tong and Leung (2002), who use behavioral assumptions to explain higher efforts by laggards.
} 
winning it, gives a player benefits later on. Clark and Nilssen (2013) interpret this as learning by doing in a two-stage contest model. Relatedly, Kovenock and Roberson (2009) and Joffrion and Parreiras (2013) model a dynamic advantage as a function of a player's past net effort, i.e., his effort over and above that of the other player. In Kovenock and Roberson's (2009) two-contest model, a high net effort in the first contest will, with some decay, give a player a head start in the second. Joffrion and Parreiras (2013) model a single all-pay auction carried out in multiple rounds, with players' effort costs in each round being convex and players observing their standings, i.e., the leader's head start, after each round. These papers consider a different strategic setting to us since, in the early contests, the margin of victory is important; our model limits the amount of advantage that can be gained from winning, and this is independent of previous efforts. A key issue in papers with effort advantage is how transferable effort is between time periods, i.e., the amount that current effort decays in future rounds. We do not have effort spillovers between contests, and the rate of catching up or lead enhancement is exogenously fixed; hence the effect of a pure win advantage can be examined.

Our analysis of the development of incentives and head starts over time is based on a stage game consisting of a complete-information two-player all-pay auction with one player having two advantages: both a head start and a higher valuation of winning. Head starts in a single contest - with and without heterogeneous valuations among the players - have been analyzed by several authors, notably by Konrad (2002), Meirowitz (2008), Kirkegaard (2012), Li and Yu (2012), Hirata (2014), Segev and Sela (2014), Siegel (2014), and Franke, et al. (2018). Among these papers, Konrad (2002) and Hirata (2014) discuss two-stage situations where players can take actions in the first stage that create head starts in the second stage, while Li and Yu (2012) and Franke, et al. (2018) discuss the principal's optimal choice of head start in a single contest in order to maximize expected effort. In contrast, we look at how head starts develop over time from an initially symmetric situation when the experience of winning gives a head start in future contests. We explore the interlinkage between the size of the head start and the derived heterogeneity in the players' valuations of winning at a certain stage contest.

The paper is organized as follows. Section 2 gives a brief analysis of a single-stage contest with a head start. Section 3 sets up the model of the sequence of contests and solves for the equilibrium. Section 4 gives an analysis of the intensity of fighting in the sequence of contests and the relative efforts of the competitors. In Section 5, we present a number of extensions to our analysis. In particular, we discuss win advantages that are combinations of head start and handicapping in Section 5.1, the effect of players' discounting future payoffs in Section 5.2, and games where stage 
prizes vary across time in Section 5.3. Section 6 offers a discussion of our main results and concludes. The proofs of our results in Sections 3 and 4 are in the Appendix, whilst the proofs for Section 5 are in an online appendix.

\section{Preliminaries: A single contest with a head start}

Before we introduce the series of contests in our main framework, it is instructive to first look at one. Consider a single all-pay auction in which two players $h$ and $k$ compete, by making irreversible efforts $x_{h}$ and $x_{k}$, over a prize of value $v_{h}=v+a$ for player $h$ and $v_{k}=v$ for player $k$, where $v>0$ and $a \geq 0$. Each player has a cost of 1 per unit effort, and efforts are translated into a score for each player in the contest by the following rule: the score of player $h$ is $z+x_{h}(z \geq 0)$, and the score of player $k$ is $x_{k}{ }^{4}$ The player with the higher score wins the contest, and ties are broken randomly with each player having probability $\frac{1}{2}$ of winning, so that the probability that player $h$ wins is

$$
p_{h}\left(x_{h}, x_{k} ; z\right)=\left\{\begin{array}{l}
1 \text { if } z+x_{h}>x_{k} \\
\frac{1}{2} \text { if } z+x_{h}=x_{k} \\
0 \text { if } z+x_{h}<x_{k}
\end{array}\right.
$$

Player $h$ is advantaged in the sense of having a head start $(z)$ and a larger value of winning the prize (by the amount $a$ ). The expected payoff of the two players is then

$$
\begin{aligned}
& \pi_{h}\left(x_{h}, x_{k} ; z\right)=p_{h}\left(x_{h}, x_{k} ; z\right)(v+a)-x_{h}, \\
& \pi_{k}\left(x_{h}, x_{k} ; z\right)=\left(1-p_{h}\left(x_{h}, x_{k} ; z\right)\right) v-x_{k} .
\end{aligned}
$$

Let $F_{h}(x)$ be the cumulative distribution function of player $h$ 's mixed strategy, i.e., $F_{h}(x)$ is the probability that player $h$ has an effort at or below $x$. $F_{k}(x)$ is defined similarly. The following Proposition characterizes the unique Nash equilibrium. ${ }^{5}$

Proposition 1 i) If $z \geq v$, then $x_{h}=x_{k}=0$.

\footnotetext{
${ }^{4}$ We can think of the players as choosing a score in the contest rather than an effort. The score cost of player $h$ is then 0 up to score $z$ and increasing per unit effort after that, whereas the score cost is linearly increasing from 0 for player $k$.

${ }^{5}$ The Proposition is proved in Clark and Riis (1995). The case of $a=0$ is proved in Konrad (2002). See also Meirowitz (2008), Hirata (2014), Siegel (2014), Franke, et al. (2018), and our online appendix.
} 
ii) If $z \in[0, v)$, then the unique mixed-strategy Nash equilibrium of the game is given by

$$
\begin{aligned}
& F_{h}\left(x_{h}\right)=\frac{z+x_{h}}{v}, \quad x_{h} \in[0, v-z] ; \\
& F_{k}\left(x_{k}\right)= \begin{cases}\frac{z+a}{v+a}, & x_{k} \in[0, z), \\
\frac{x_{k}+a}{v+a}, & x_{k} \in[z, v] .\end{cases}
\end{aligned}
$$

In this equilibrium, the expected efforts of the players are

$$
x_{h}^{*}(z, a)=\frac{(v-z)^{2}}{2 v}, \text { and } x_{k}^{*}(z, a)=\frac{v^{2}-z^{2}}{2(v+a)} ;
$$

expected payoffs are

$$
\pi_{h}^{*}(z, a)=z+a, \text { and } \pi_{k}^{*}(z, a)=0 ;
$$

and probabilities of winning are

$$
\begin{aligned}
& p_{h}^{*}(z, a)=1-\frac{v^{2}-z^{2}}{2 v(v+a)}, \\
& p_{k}^{*}(z, a)=\frac{v^{2}-z^{2}}{2 v(v+a)} .
\end{aligned}
$$

Note that the expected payoff of the disadvantaged player is $p_{k}^{*} v-x_{k}^{*}=$ 0 . Hence, the probability that he wins follows his expected effort closely: $p_{k}^{*}=\frac{x_{k}^{*}}{v} \cdot{ }^{6}$

We see from (5) that the advantaged player has a larger expected payoff from the contest. He has the larger probability of winning in equilibrium since he starts with a positive score at no cost. It can easily be verified that $p_{h}^{*}(z, a) \geq p_{k}^{*}(z, a)$, with equality only if $z=a=0$. Note also that the support of the distribution of effort for player $h$ is $[0, v-z]$, whereas the support for the corresponding distribution of $h$ 's contest score is $[z, v]$.

In what follows, we shall be interested in the relative efforts of the players, rather than the contest score per se. We see from (2) and (3) that the disadvantaged player $k$ on one hand has a higher probability of being inactive but that he, conditional on being active, exerts a higher expected effort. This translates, by way of (4), into the following:

Corollary 1 The disadvantaged player exerts the larger expected effort of the two if and only if

$$
a<\frac{2 v z}{v-z} .
$$

\footnotetext{
${ }^{6}$ Note that, although (1) includes the possibility of a tie occurring, this will not happen in equilibrium. Player $h$ randomizes continuously on $[0, v-z]$, and the only atom in player $k$ 's strategy is isolated at a level at least $z$ below the score of the opponent; otherwise, player $k$ randomizes continuously over the interval $[z, v]$. Hence the highest score is unique with probability 1 , and a tie will not occur.
} 
This says that the laggard is expected to have more effort than his rival when his disadvantage in terms of the value of winning is sufficiently weak relative to the prize and the leader's head start. This is evident from (2) and (3): whereas $v$ and $z$ affect the efforts of both players, $a$ affects the disadvantaged player's effort only - the more disadvantaged he is in terms of the value of winning, the higher is the probability that he is inactive. Note that, if the players have the same value of winning the prize $(a=0)$, then the disadvantaged player has greater effort in expectation. This is a feature of the final contest in the sequence of $T$ that we consider below.

These results are used in the next sections to solve and analyze our model.

\section{Sequential contests}

There are two identical players, $i=1,2$, who compete in a series of $T \geq 2$ all-pay auctions for a prize of $v$ in each contest by making irreversible efforts $x_{i, t} \geq 0, t=1, \ldots, T$.

The probability of winning for player 1 in contest $t$ depends on current effort as well as on the history so far, summarized by the net number of wins that player 1 has in the previous $t-1$ contests. Denote this by $M_{t}$. Suppose in particular that at contest $t$, player 1 has won $m_{t}$ of the $t-1$ past contests, and player 2 has won $\left(t-1-m_{t}\right)$. Hence $M_{t}:=m_{t}-\left(t-1-m_{t}\right)=2 m_{t}-t+1$. Without loss of generality, we shall assume that $M_{t} \geq 0$; i.e., if the game has a leader, this is always player $1 .^{7}$

The parameter $M_{t}$ represents a head start, making it possible for player 1 to win the current contest with less effort. In particular, the score for player 1 in contest $t$ is given by the sum of his current effort $x_{1, t}$ and his head start. The actual size of the head start depends on the net number of wins, and also on how much this parameter influences the score of the player in the contest. We model the head start in contest $t$ as $M_{t} s$ where $s$ is the size of the advantage from winning one previous contest. Hence the score of player 1 in contest $t$ is $M_{t} s+x_{1, t}$, and that of player 2 is $x_{2, t}$. To ensure that no subgame can occur in which no effort is exerted, we assume that $^{8}$

$$
s \in\left(0, \frac{v}{T-1}\right)
$$

\footnotetext{
${ }^{7}$ This is without loss of generality since the case where player 2 leads $\left(M_{t} \leq-1\right)$ can be analyzed by taking the absolute value of $M_{t}$ and interchanging the players.

${ }^{8}$ See Section 5.3 for a discussion of a case where this restriction is relaxed.
} 
The probability that player 1 wins contest $t$ is

$$
p_{1, t}\left(x_{1, t}, x_{2, t}\right)=\left\{\begin{array}{l}
1 \text { if } M_{t} s+x_{1, t}>x_{2, t} \\
\frac{1}{2} \text { if } M_{t} s+x_{1, t}=x_{2, t} \\
0 \text { if } M_{t} s+x_{1, t}<x_{2, t}
\end{array}\right.
$$

At contest $t$, the maximum number of net wins for player 1 is $t-1$, meaning that he has won all the previous $t-1$ contests. If player 1 has won all but one of the previous $t-1$ contests, then his net win advantage is $t-3$, whereas the net win advantage is $t-5$ if player 1 has won all but two of the previous contests, and so on.

Note the parallel to the single contest discussed in Section 2: we can write $M_{t} s=z_{t}$ as the head start in contest $t$. Below we show that the head start conveys an extra incremental value to the leader over and above the value of losing in the continuation of the game, and this will be represented by $a_{t}$. In the one-shot version of the game, these parameters are independent, but we reveal below that they are interlinked in the dynamic model; hence, a head start in a dynamic model can influence play through more channels than in the one-shot case.

Denote by $u_{i, t}^{*}\left(M_{t}\right)$ the value for player $i$ of the subgame starting at contest $t$ with a net number of wins for player 1 equal to $M_{t}$. Referring back to the notation introduced in equation (5) above, we can write

$$
u_{1, t}^{*}\left(M_{t}\right):=\pi_{1}^{*}\left(M_{t} s, a_{t}\right) .
$$

For $M_{t} \geq 1$, we know from Proposition 1 that $u_{1, t}^{*}\left(M_{t}\right)=M_{t} s+a_{t}$ and $u_{2, t}^{*}\left(M_{t}\right)=0$. Crucial for solving the game with $T$ sequential contests is finding an expression for $a_{t}$.

For ease of notation, we denote in the following, for each contest $t$, the expected effort of player $i$ in that contest by $x_{i, t}^{*}\left(M_{t}\right)$, and total expected effort by $X_{t}^{*}\left(M_{t}\right):=x_{1, t}^{*}\left(M_{t}\right)+x_{2, t}^{*}\left(M_{t}\right)$.

Consider the final contest $T$. Since there are no future prizes, $a_{T}=$ 0 , and by definition $z_{T}=M_{T} s$. Thus, expected efforts and payoffs in equilibrium are

$$
\begin{aligned}
& x_{1, T}^{*}\left(M_{T}\right)=\frac{\left(v-M_{T} s\right)^{2}}{2 v}, \quad x_{2, T}^{*}\left(M_{T}\right)=\frac{v^{2}-\left(M_{T} s\right)^{2}}{2 v} ; \\
& u_{1, T}^{*}\left(M_{T}\right)=M_{T} s, \quad u_{2, T}^{*}\left(M_{T}\right)=0 .
\end{aligned}
$$

Note that, from (9) - and in line with Corollary 1 - we can state the following:

Corollary 2 Player 2 (the laggard) has the higher expected effort in the last contest for any $M_{T} \geq 1$. 
If $M_{T}=0$, so that each player has won equally many of the previous contests, then the game in this last contest is symmetric and we have

$$
\begin{aligned}
& x_{1, T}^{*}\left(M_{T}=0\right)=x_{2, T}^{*}\left(M_{T}=0\right)=\frac{v}{2} ; \\
& u_{1, T}^{*}\left(M_{T}=0\right)=u_{2, T}^{*}\left(M_{T}=0\right)=0 .
\end{aligned}
$$

Total expected effort in contest $T$ is

$$
X_{T}^{*}\left(M_{T}\right)=v-M_{T} s
$$

Consider next any contest $t \in\{2, \ldots, T-1\}$ in which $M_{t} \geq 1$, i.e., player 1 has at least one more win than player 2 so far. Denote the mixed strategy of player $i$ in contest $t$ by $F_{i, t}(x)$, so that the expected payoff for player 1 is

$$
\begin{aligned}
u_{1, t}\left(x_{1, t}, M_{t}\right): & =F_{2, t}\left(x_{1, t}+M_{t} s\right)\left[v+u_{1, t+1}^{*}\left(M_{t}+1\right)\right] \\
& +\left[1-F_{2, t}\left(x_{1, t}+M_{t} s\right)\right] u_{1, t+1}^{*}\left(M_{t}-1\right)-x_{1, t} .
\end{aligned}
$$

That is, either he wins, receives the prize $v$ for this contest, increases his net number of wins by one, and gets a continuation payoff $u_{1, t+1}^{*}\left(M_{t}+1\right)$; or he loses, receives no prize in the current contest, decreases his net number of wins by one, and gets a continuation value of $u_{1, t+1}^{*}\left(M_{t}-1\right)$. Quite straightforwardly, we can rewrite this as

$$
u_{1, t}\left(x_{1, t}, M_{t}\right)=u_{1, t+1}^{*}\left(M_{t}-1\right)+F_{2, t}\left(x_{1, t}+M_{t} s\right)\left(v+a_{t}\right)-x_{1, t},
$$

where

$$
a_{t}:=u_{1, t+1}^{*}\left(M_{t}+1\right)-u_{1, t+1}^{*}\left(M_{t}-1\right) .
$$

Note that, if $M_{t}=1$, then $u_{1, t+1}^{*}\left(M_{t}-1\right)=0$, since contest $t+1$ becomes symmetric if the advantaged player 1 loses contest $t$ in this case.

Player 2 is at a disadvantage, being at least one net win down. If he wins the current contest, then he gains the stage prize $v$. But even with a win, he will continue as the disadvantaged player earning zero, or at best - if winning at $M_{t}=1$ - getting even, but still earning zero. Thus, the expected payoff to player 2 is given by

$$
u_{2, t}\left(x_{2, t}, M_{t}\right):=F_{1, t}\left(x_{2, t}-M_{t} s\right) v-x_{2, t} .
$$

If $M_{t}=0$, then the game is symmetric; the winner of contest $t$ gets the stage prize and the continuation from being the leader, and the loser gets nothing since the continuation payoff of losing from this state is 0 . The expression for player $i$ 's expected payoff is then

$$
u_{i, t}\left(x_{i, t}, 0\right):=F_{j, t}\left(x_{i, t}\right)\left[v+u_{1, t+1}^{*}(1)\right]-x_{i, t}
$$


In this case, the contest is symmetric over a prize of $v+u_{1, t+1}^{*}(1)$ for each player, and each player has an expected effort of

$$
\frac{1}{2}\left[v+u_{1, t+1}^{*}(1)\right]
$$

with an expected payoff of 0 . Since, by definition, $M_{1}=0$, this analysis holds for the first contest at $t=1$.

Proposition 2 summarizes the equilibrium expected efforts and expected payoffs of the $T$ sequential contests. The proof is in the Appendix.

Proposition 2 In a contest $t \in\{2, \ldots, T\}$ with $M_{t} \geq 1$, equilibrium expected efforts of the players are

$$
\begin{aligned}
x_{1, t}^{*}\left(M_{t}\right) & =\frac{\left(v-M_{t} s\right)^{2}}{2 v}, \\
x_{2, t}^{*}\left(M_{t}\right) & =\frac{v^{2}-M_{t}^{2} s^{2}}{2[v+2 s(T-t)]} ;
\end{aligned}
$$

with win probabilities

$$
\begin{aligned}
p_{1, t}^{*}\left(M_{t}\right) & =1-\frac{v^{2}-M_{t}^{2} s^{2}}{2 v[v+2 s(T-t)]} \\
p_{2, t}^{*}\left(M_{t}\right) & =\frac{v^{2}-M_{t}^{2} s^{2}}{2 v[v+2 s(T-t)]} .
\end{aligned}
$$

and equilibrium expected payoffs

$$
\begin{aligned}
u_{1, t}^{*}\left(M_{t}\right) & =s(T-t+1)\left[M_{t}+\frac{1}{2}(T-t)\right], \\
u_{2, t}^{*}\left(M_{t}\right) & =0 .
\end{aligned}
$$

In a contest $t$ with $M_{t}=0$, including contest 1 , equilibrium expected efforts, win probabilities, and payoffs are

$$
\begin{aligned}
x_{i, t}^{*}(0) & =\frac{1}{2}\left[v+\frac{1}{2} s(T-t)(T-t+1)\right] ; \\
p_{i, t}^{*}(0) & =\frac{1}{2} \\
u_{i, t}^{*}(0) & =0 ; \quad i=1,2 .
\end{aligned}
$$

Note, from (20), that there is a hard fight to win the first contest, where total expected effort is $v+\frac{1}{2} s T(T-1)$.

It is straightforward to show that the probability that the laggard wins a stage contest in (18) is always less than one half; furthermore, the laggard's 
probability of winning is lower, the more he is lagging, i.e., the higher is $M_{t}$; and the longer is the rest of the game, i.e., the higher is $(T-t)$.

Two factors play a role in determining the course of play: (i) the head start that affects the contest score of the advantaged player, $z_{t}=M_{t} s$; and (ii) the difference $a_{t}$ in the value of winning and losing for the advantaged player. As shown in the proof of Proposition 2 in the Appendix, the latter equals

$$
a_{t}=2 s(T-t) .
$$

Note that this value does not depend on how big the lead of player 1 is, i.e., on $M_{t}$, nor on the size of the stage prize $v$. This latter independence can be seen from Proposition 1, where payoffs in the stage contest are determined purely by the head start and the extra valuation of the leader. In our dynamic model, this manifests itself in (19) which is independent of $v$. Since the expected payoff at each stage can be written in this way, the difference between winning and losing at any stage is independent of the stage prize. The fact that $a_{t}$ is independent of $M_{t}$ follows from (19), which is linear in $M_{t}$, combined with the definition of $a_{t}$. The continuation value increases in both the number of contests remaining after contest $t$ is played (i.e., $T-t$ ) and the win advantage $s$. Whereas an increase in the head start $z_{t}$ decreases the expected efforts of both players, increasing the value of $a_{t}$ only affects the expected effort of the laggard, and negatively so, according to Proposition 1 . Hence, the lead in contest $t$, measured by $M_{t}$, reduces the expected effort of both players while the leader's difference in continuation payoff only reduces the effort of the laggard. Equation (23) captures a subtle interplay between the head start parameter $s$ and the implied difference in the leader's valuations of winning and losing.

The expected payoff of the advantaged player at contest $t$ has a simple form, as indicated by (19). In this expression, $T-t+1$ is the number of contests remaining when we reach contest $t$. Hence, the expected equilibrium payoff to the player with a head start is conveniently expressed as a function of the number of remaining contests, the number of net wins, and the size of the advantage per win.

In what follows, we shall concentrate the analysis on the expected efforts of the players. It is, however, worth noting that the scores of the players determine the contest outcome at each stage. Conditional on player 1 choosing positive effort, his effort comes from a uniform distribution over the interval $\left(0, v-M_{t} s\right]$, which becomes more narrow as the head start increases, and his score comes from a uniform distribution over the interval $\left(M_{t} s, v\right]$. Likewise, conditional on positive effort by player 2, his effort (and score) comes from a uniform distribution on $\left(M_{t} s, v\right]$ which also narrows as player 1's head start increases. It follows that, conditional on positive efforts, the two players' scores have the same distribution. Still, considering the mass points (player 1's point is higher and has lower mass), the expected 
score of player 1 is the higher one, as can be seen from equations (15) and (16):

$$
\begin{aligned}
z_{t}+x_{1, t}^{*}\left(M_{t}\right) & =M_{t} s+\frac{\left(v-M_{t} s\right)^{2}}{2 v} \\
& =\frac{v^{2}-M_{t}^{2} s^{2}}{2 v}>\frac{v^{2}-M_{t}^{2} s^{2}}{2[v+2 s(T-t)]}=x_{2, t}^{*}\left(M_{t}\right) .
\end{aligned}
$$

\section{$4 \quad$ Keep on fighting}

The amount of fighting (effort) expected in equilibrium is simple to calculate; the expected payoff in equilibrium for the game as a whole is zero, so that the players compete away the whole surplus. This leads to the following Corollary to Proposition 2.

Corollary 3 Total expected effort over the $T$ contests is $v T$.

Given this result, we are interested in how the intensity of effort varies across contests, depending on the net number of wins, and on whether the result of the current contest makes the subsequent one more or less even. In a one-shot symmetric all-pay auction, total expected effort equals the prize, here $v$. This is not so in our model. A contest with symmetry, i.e., where $M_{t}=0$, is necessarily followed by a contest where one player has a head start, since one player evidently will be declared the winner of contest $t$ and thus obtain a net win of 1 . We find that the total expected effort at symmetry is larger than the stage prize but that it is bound to fall in the subsequent contest when one player has achieved a head start. Recalling that $X_{t}^{*}\left(M_{t}\right)$ is total expected effort in contest $t$, we have the following, with proof in the Appendix.

Corollary 4 For $t \in\{1, \ldots, T-1\}$, we have (i) $X_{t}^{*}(0)>v$; and (ii) $X_{t}^{*}(1)<v-s$.

Part (i) indicates the intensity of fighting. Note that it is the anticipation of receiving a head start that drives the extra effort; one-shot contests cannot of course capture such a phenomenon. Part (ii) shows that the intensity of fighting falls in the subsequent contest, where $M_{t}=1$. Actually, there can be symmetry only in odd-numbered contests: It is only when $t-1$ is even that the gross number of previous wins can be the same for the two players at contest $t$ so that symmetry entails.

As we advance through the series of contests, symmetry yields less expected efforts in sum. This is seen directly from (20), which is decreasing in $t$. We have: 
Corollary 5 Suppose there is symmetry in some contest $t \in\{1, \ldots, T\}$, i.e., $M_{t}=0$. Then $X_{t}^{*}(0)$ is lower, the higher is $t$.

Intuitively, the less future there is after a contest, the less value there is to becoming the leader from the symmetric state.

We turn next to contests in which $M_{t}>0$, where we are interested in the extent to which a laggard will keep on fighting, even though the opponent has a head start. Using Proposition 2 together with Corollary 1, we have a central result in this respect:

Corollary 6 In any contest $t \geq 2$ where $M_{t} \geq 1$, the laggard has higher expected effort than the leader if and only if

$$
T-t<\frac{v M_{t}}{v-M_{t} s}
$$

From this we can conclude that the laggard in expectation has more effort than the leader in cases where: there are a low number of contests left (low $T-t$ ); he is at a large disadvantage (large $M_{t}$ ); the head start parameter $(s)$ is high; and the stage prize $v$ is low.

These results reflect the findings in Section 2 above. When there are relatively few contests left, the difference in valuation between winning and losing, $a_{t}$, becomes small. The value of $a_{t}$ affects the laggard's effort negatively but does not affect the leader's effort, whereas the head start $M_{t}$ affects both efforts negatively. It can easily be verified that the negative effect that increasing $M_{t}$ has on the leader's effort is larger in magnitude than the reduction in that of the laggard. Hence the leader slacks off by more than the laggard is discouraged following an increase in the net wins. The role of $s$ is more subtle since it works through two channels. It affects the score of the leader in the current contest directly, causing less effort by both competitors; at the same time it increases $a_{t}$, which reduces only the laggard's effort. The larger is $s$, the more $a_{t}$ falls in each successive contest, which raises the effort of the laggard. Hence, although increases in $M_{t}$ and $s$ both lead to a higher likelihood that the laggard will have more effort, they work through different channels. Again, the interplay between the head start and the leader's difference in continuation payoffs from winning and losing is apparent.

Our results are partly driven by the fact that competitors can win a prize at each stage. ${ }^{9}$ The comparative-static properties of (15) and (16) show that an increase in $v$ will tend to raise the expected effort of the

\footnotetext{
${ }^{9}$ Strong discouragement effects for the laggard are typical of races without stage prizes. Introducing a stage prize in a race mitigates the discouragement effect; see Konrad and Kovenock (2009), a paper which is discussed in more detail in Section 6 below.
} 
leader relative to the laggard when there are many contests left, and that the laggard's effort will be raised the most in later stages of the contest. Early in the series of contests, a leader has a great deal to fight for since $a_{t}=2 s(T-t)$ is large. Increasing $v$ strengthens this effect. Later on, $a_{t}$ falls, giving the laggard relatively more to fight for.

Now we consider the laggard's probability of winning a contest. Even if the laggard eventually has the higher effort, his probability of winning is always smaller, as we have seen. This mirrors the result from the one-shot contest. However, we can show that the laggard's probability of winning increases in the contest after a win, and may increase even after a loss. In particular, we have the following:

Corollary 7 Consider a contest $t \in\{2, \ldots, T-1\}$ where $M_{t} \geq 1$. Player 2 's probability of winning increases from contest $t$ to contest $t+1$ if he wins contest $t$. It increases even after a loss in contest $t$, if

$$
\frac{v}{s}>\frac{1}{4}\left\{2 M_{t}+1+\sqrt{[16(T-t)+1]\left(2 M_{t}+1\right)+2 M_{t}\left(10 M_{t}+1\right)}\right\} .
$$

After a win by the lagging player 2 , both $M_{t}$ and $(T-t)$ go down, and so surely his probability of winning gets higher. But this can also happen after a loss where player 1's head start is increased to $M_{t}+1$. Then player 2 's probability of winning contest $t+1$ is

$$
\frac{v^{2}-\left(M_{t}+1\right)^{2} s^{2}}{2 v[v+2 s(T-t-1)]} .
$$

Comparing this and (18), we obtain the result in Corollary 7 . We see that such an increase in the probability of winning occurs when the stage prize $v$ is sufficiently high relative to $s, M_{t}$, and $(T-t)$. Low $s$ and/or $M_{t}$ means that the head start changes quite little after the laggard has lost a contest (implying that he has relatively little to make up). When the number of remaining contests is low, we know that this incites the laggard to effort, as does a higher stage prize (since this is the only prize he has to fight for).

We can use Corollary 6 to discuss under which circumstances a laggard will have the higher expected effort. Consider first short contests, where $T \in\{2,3\}$. When $T=2$, Corollary 2 indicates that the laggard will have higher expected effort in the final contest. For $T=3$ we have the following result:

Corollary 8 When $T=3$, the expected effort of the laggard is larger than the leader at $t=2$.

When the series consists of three contests, the laggard will always have more expected effort than the leader in the second contest, and also in the 
final one, should he still be disadvantaged at this stage. From (15) and (16), it can be verified that the net win advantage, as measured by $M_{t}$, reduces the expected effort of the leader by more than the laggard. Modifying this effect is the fact that the winner of the first contest has more to fight for, as measured by $a_{2}$, which is zero when $T=2$ and $2 s$ when $T=3$. Hence, there is no effect on the expected effort of the laggard through this channel in the former case, and a negative effect in the latter. In sum, however, the expected effort of the leader falls more in such short series of contests.

The following Proposition sums up results on how the relative expected efforts of leader and laggard develop for games of four rounds or more; the proof is in the Appendix.

Proposition 3 (i) Let $T \geq 4$. For some contest $t \in\{2, \ldots, T-1\}$, there is a leader who has lower expected effort than the laggard, i.e., if player 1 is that leader so that $M_{t} \geq 1$, then we have $x_{2, t}^{*}\left(M_{t}\right)>x_{1, t}^{*}\left(M_{t}\right)$ for that contest.

(ii) Let $T \geq 4$. For $t \in\{2, . ., T-1\}$ and $M_{t} \geq 1$, suppose that $x_{2, t}^{*}\left(M_{t}\right)>x_{1, t}^{*}\left(M_{t}\right)$. Then $x_{2, t+1}^{*}\left(M_{t}+1\right)>x_{1, t+1}^{*}\left(M_{t}+1\right)$.

(iii) Let $T \geq 5$. For $t \in\{3, \ldots, T-2\}$ and $M_{t} \geq 2$, suppose that $x_{1, t}^{*}\left(M_{t}\right)>x_{2, t}^{*}\left(M_{t}\right)$. Then $x_{1, t+1}^{*}\left(M_{t}-1\right)>x_{2, t+1}^{*}\left(M_{t}-1\right)$.

(iv) Let $T \geq 5$. For $t \in\{3, \ldots, T-2\}$ and $M_{t} \geq 2$, suppose that $x_{2, t}^{*}\left(M_{t}\right)>x_{1, t}^{*}\left(M_{t}\right)$. Then $x_{1, t+1}^{*}\left(M_{t}-1\right)>x_{2, t+1}^{*}\left(M_{t}-1\right)$ is possible.

Part (i) of this Proposition is an important result. At some contest before the final one, the expected effort of a laggard will always be larger than that of the advantaged player. In this sense the laggard does indeed "keep on fighting". The intuition is based upon the combination of two effects discussed previously: the head start which reduces both efforts, and that of the laggard more, and the reduction in the difference in the continuation payoffs from winning and losing for the leader in the series, which encourages the laggard as the game progresses. Eventually, the latter effect is the stronger, and the laggard has the higher expected effort.

Part (ii) depicts an enduring laggard in the sense of not giving up the fight. If at contest $t$ he has more expected effort but loses, then his response will be to have more expected effort than the leader also in the following contest. The transition from contest $t$ to contest $t+1$ here implies an increased head start for the leader causing him to slack off more, while the progression of the contest lowers the difference in the continuation values of the leader.

Part (iii) can be thought of as an enduring leader continuing to exert more expected effort than the opponent if he should lose a contest but still have a head start. In this case, the transition of the contest from $t$ to $t+1$ implies a smaller head start; both expected efforts increase, affecting the leader more. 
Part (iv) demonstrates the possibility that success may "discourage" the laggard. Here the laggard has the higher expected effort in a contest; if he wins the contest and is still disadvantaged, then it is possible that he will exert less effort in expectation than the leader in the next contest.

Parts (ii) and (iii) of Proposition 3 can be combined to show that the sign of the difference in efforts of the players is invariant to loss in the following sense:

Corollary 9 Suppose $T \geq 5$ and $M_{t} \geq 2$. Irrespective of who has the greater expected effort in contest $t \in\{3, \ldots, T-2\}$, if this player loses that contest, then he will have greater expected effort also in contest $t+1$.

\section{Extensions}

In this Section, we discuss three departures from the basic model. In Section 5.1, we allow the win advantage to materialize as a combination of head start and handicapping, thus departing from the contest success function in (8). In Section 5.2, we discuss how the equilibrium would be affected by players discounting future payoffs. In Section 5.3, we study a sequence of all-pay auctions where prizes vary across time. Proofs for this Section are contained in the online appendix.

\subsection{Head start vs handicapping}

In our main analysis, the effect of a win in today's contest is to create a head start for the winner in future contests. It can be argued that this is a narrow view of such a win advantage. An alternative is to allow for the win advantage to take the form in part of a head start for the winner and in part of a handicap for the loser. ${ }^{10}$ In order to model this, let us replace the contest success function in (8) with the following:

$$
p_{1}\left(x_{1}, x_{2}\right)=\left\{\begin{array}{l}
1 \text { if } b M_{t} s+x_{1, t}>\left[1-(1-b) M_{t} s\right] x_{2, t} \\
\frac{1}{2} \text { if } b M_{t} s+x_{1, t}=\left[1-(1-b) M_{t} s\right] x_{2, t} \\
0 \text { if } b M_{t} s+x_{1, t}<\left[1-(1-b) M_{t} s\right] x_{2, t}
\end{array}\right.
$$

where $b \in[0,1]$. This case can be viewed as giving the win advantage both an additive component, on the left hand side of (25), and a multiplicative component, on the right hand side. In the terminology of Konrad (2002), such an additive advantage is a head start for player 1 , while the multiplicative disadvantage is a handicap for player 2 . This set-up collapses to

\footnotetext{
${ }^{10}$ See Konrad (2002), Kirkegaard (2012), and Franke et al. (2018) for analyses of a single all-pay auction with both a head start and a handicap.
} 
our earlier case when $b=1$. The higher is $b$, the more of the win advantage comes as a head start and correspondingly less as a handicap.

We impose the following restriction on parameters:

$$
s(T-1)<\frac{v}{b+v(1-b)},
$$

which is a modification of $(7)$ to the present case. Note that, for $b<1$, (26) is stricter than (7) if and only if $v>1$, and that it reduces to (7) when $b=1$. With this restriction, we can carry out an analysis parallel to the one above; see the online appendix for details. That analysis indicates that handicapping discourages the laggard when $v$ is large. In particular, when $v>\frac{b}{1-b}$, the opposite of Corollary 8 is true: the leader exerts the higher expected efforts at $t=2$ when $T=3$.

\subsection{Discounting}

We have so far simplified the analysis by disregarding players' discounting of future payoffs. Suppose, alternatively, that the players use a common discount factor $\delta \in(0,1]$. The difference for the leader from winning and losing contest $t$ now is

$$
a_{t}=2 s \frac{1-\delta^{T-t}}{1-\delta}
$$

which is increasing in $\delta$ for $t \leq T-2$ and approaches $2 s(T-t)$ as $\delta$ approaches 1; see the online appendix for this result, as well as further elaborations. With discounting, Corollary 6 is modified, in that the condition in (24) becomes

$$
\frac{1-\delta^{T-t}}{1-\delta}<\frac{v M_{t}}{v-M_{t} s}
$$

Thus, we can add heavy discounting to the factors, discussed in Section 4, that lead to the laggard having a higher expected effort than the leader.

\subsection{Varying prizes}

In the main analysis, we assume that there is a prize of value $v$ in each contest. Allowing this prize to vary across the contests does not have too strong an effect on the outcome of the game as long as the contest prize in each contest, denoted $v_{t}$, adheres to a weakened version of condition (7), namely that, for each contest $t, v_{t} \geq s(t-1)$. When this does not hold, there will be cases where the leader's lead will be so great that the laggard concedes and the players exert no effort at all in one or more of the contests, in line with part (i) of Proposition 1.

In order to explore the possible outcomes when prizes vary, consider the case of $T=3$. Let $v_{t} \geq 0$ be the prize in contest $t \in\{1,2,3\}$. Suppose 


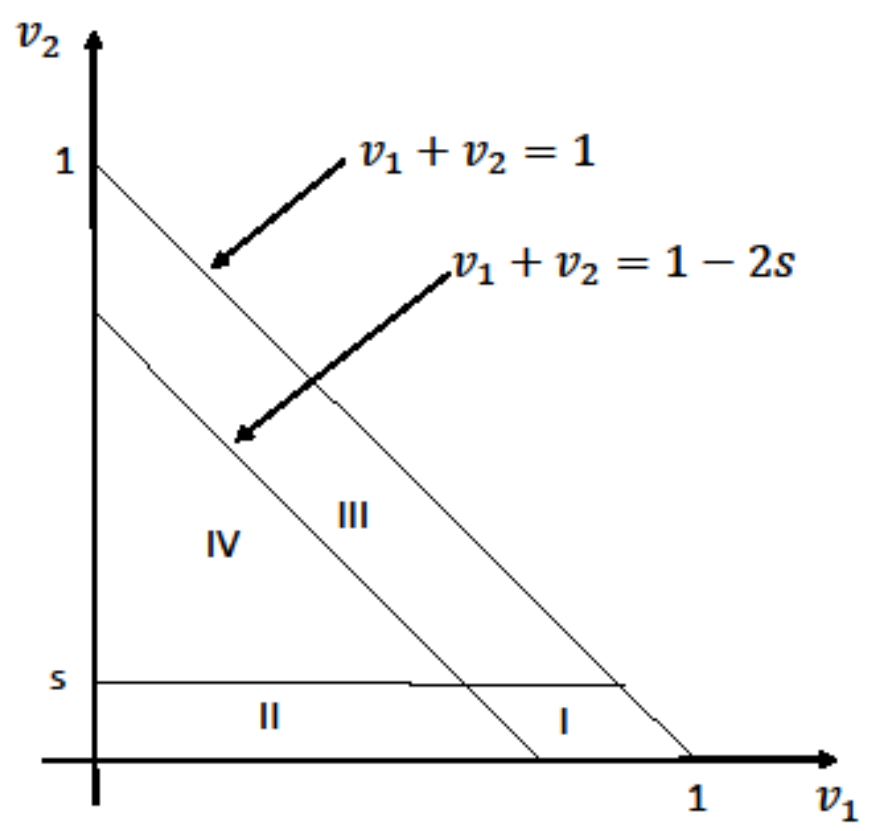

Figure 1: Varying prizes.

the contest designer has a total budget of 1 to spend in total in the three contests, so that $v_{1}+v_{2}+v_{3}=1$, implying $v_{3}=1-v_{1}-v_{2}$, and assume that $s \in\left(0, \frac{1}{6}\right)$.

The equilibrium outcome of this game is illustrated in Figure 1, which describes the distribution of prizes in $\left(v_{1}, v_{2}\right)$ space; given the fixed total prize budget, the third prize, $v_{3}=1-v_{1}-v_{2}$, is measured by the distance from the $v_{1}+v_{2}=1$ line. Details of the analysis of this case are in the online appendix.

We can delineate four areas in Figure 1 in which the game is played out differently. If $1-v_{1}-2 s \leq v_{2} \leq s$, so that we are in area I of Figure 1, then both $v_{2}$ and $v_{3}$ are so small, relative to the win advantage $s$, that the player losing contest 1 does not consider them worth fighting for, and there are no efforts in contests 2 and 3.

If $v_{2}<1-v_{1}-2 s$ at the same time as $v_{2} \leq s$, so that we are in area II in Figure 1, then there are no efforts in contest 2. Efforts are exerted in contest 1, mainly in order to obtain the win advantage and get in position before the showdown in contest 3 , where the big prize is.

If $v_{2} \geq 1-v_{1}-2 s$, as well as $v_{2}>s$, so that we are in area III in Figure 1 , then there is no effort in contest 3 in the case of one player winning both contests 1 and 2. Now, $v_{2}$ is big enough for there to be something to fight for in contest 2 , while $v_{3}$ is so small that the laggard's incentives disappear in the event of a second loss.

The case of $s<v_{2}<1-v_{1}-2 s$ corresponds to area IV in Figure 1 
and covers that of equal stage prizes (here $v_{1}=v_{2}=v_{3}=\frac{1}{3}$ ) discussed in the main analysis. Now, both $v_{2}$ and $v_{3}$ are large enough that a player has incentives to stay in the game throughout, even if he should lose both contest 1 and contest 2 .

In summary, we find that the outcome of the game that we have discussed in our main analysis is relatively robust to variations in prizes, as long as late prizes do not become too small. In particular, the assumption in (7) can be replaced with the weaker condition $s(t-1)<v_{t}$, for each $t$. Thus, for example, any $v_{1}>0$ in the first contest can be allowed.

\section{Discussion and conclusion}

In this paper we have examined a finite series of all-pay auctions that are linked through time. Specifically, a player who has won more contests than he has lost is assumed to build up a win advantage in the form of a head start over the rival, and the more net wins the larger the advantage. This way, we endogenize head starts and explain them as outcomes of previous contests with win advantages. The effect captured here may be purely psychological or experience-based, but may also be due to factors such as sellers who gain more back-room resources, or researchers who get more assistants. The series of contests has a symmetric outset, and we identify effects overlooked in static contest models. Two effects are at work that influence efforts of leaders and laggards. First, a head start leads both players to exert lower effort in expectation, but affects the laggard most; exerting effort will at best even up the contest, at which point both players will expend many resources to gain the lead. Second, the head start creates an extra value to the leader by ensuring easier access to future prizes, hence reducing the effort of the laggard further. The relative magnitude of these effects changes throughout the series of contests, however, so that, eventually, the laggard has the higher expected effort. We have also investigated the subtle relationship between the size of the head start and the difference in the continuation value for the leader from winning and losing.

Series of contests can be combined in a plethora of ways. It is instructive to compare our results with those of a race considered by Konrad and Kovenock (2009). They present a rich model with heterogeneous contestants, but also allude to the symmetric version as an important special case. By factoring out the initial asymmetry between players, it is possible to uncover the underlying mechanisms which drive behavior in our and their analysis. The version of their model that is most comparable is one in which a stage prize $v$ is on offer in each contest, and the first player to cross the finish line is also rewarded with $v$. Two players compete in an all-pay auction, and the state describes how far away each player is from 
the finishing line.

In the symmetric version of their model, three scenarios are possible. 1) When one player has a large lead, the stage contest is one in which each player fights for the stage prize $v$, exerting equal effort in expectation, and competing away the value of the stage prize. 2) In a symmetric state, the stage contest is an all-pay auction where each player fights for a prize of $2 v$ (the value of the current prize, and getting a lead for the prize in the final contest). Intense fighting ensues and the value of the prize to the players at this stage is dissipated in expectation. This behavior occurs independently of whether the symmetric state is close to or far from the finishing line. 3) The third type of stage contest is asymmetric in the sense that the leader has a larger stake than the laggard. This can occur in a state directly previous to and directly after symmetry. A leader who sees that his lead can be eradicated (by reaching the symmetric state) has a stake of $2 v$ (the value of the stage prize and remaining the leader); the laggard has only a value of $v$, the current stage prize, since equaling the score leads to symmetry where rents are completely dissipated. Here, the leader fights harder than the laggard to retain the lead. A head start in this model can be considered a starting point at which one player has fewer steps to the finishing line. If the head start is one step, then there is low intensity of fighting, and the leader has the larger expected effort (scenario 3 ). If the head start is larger than this, then the whole of the stage prize is dissipated (scenario 1).

Our results contrast with those of Konrad and Kovenock (2009) in several ways. In the symmetric version of their model, the laggard will never have a greater expected effort than the leader, whereas demonstrating this possibility in our model has been a main focus. Only one type of stage contest exhibits asymmetry (scenario 3), and here the leader has the larger prize, larger expected effort and larger probability of winning. Hence, our results from Corollary 7 and Proposition 3 have no parallel in the Konrad and Kovenock analysis. In scenario 2 above, the players' efforts sum to more than the value of the stage prize which is an important facet of dynamic competition. Efforts at each stage are determined by the value of the stage prize and the players' continuation payoffs from that point. The amount that expected effort in scenario 2 is larger than the stage prize is constant, and not dependent upon the size of the lead, or number of steps left to the finish line. Our model also exhibits contests in which expected effort is larger than the value of the stage prize in symmetric states (Corollary 4), but the amount of expected effort is largest at the outset as the players compete for an initial advantage, falling monotonically at later symmetric states (Corollary 5). Indeed, the amount of expected efforts in relation to the stage prize can be large in our model, since there is a large value to being the initial leader: considering the first contest, and 
setting $s$ as large as possible from (7), one can use (20) to calculate that the maximal expected effort approaches $v\left(1+\frac{T}{2}\right)$, compared to $2 v$ in Konrad and Kovenock (2009). In our model, it may be the contest score that is observable and not efforts per se. Since the score is a player's effort plus the previous number of wins, it may appear that fighting is more intense than is actually the case.

The force that drives the differences in the models is the exact nature of the connection between stage contests in the two analyses. In Konrad and Kovenock (2009), players advance through a grid of states as the play progresses and players approach the finishing line. At any current state, the players may have a symmetric valuation of the continuation of the game, or they may have asymmetric valuations (scenario 3). There are no connections between contests other than the effect on the valuations at a stage contest. From our Proposition 1, one can see that this reduces the expected effort of the laggard only. In our case, the amount that the leader values winning over losing in a stage contest is independent of how many net wins he has, as depicted by $a_{t}$, which is independent of $M_{t}$. This parallels Konrad and Kovenock (2009) and is a feature of the linearity in the payoff functions in each model. In our model, the history - as captured by the net number of wins - determines the head start of the leader. In modelling terms, this means that the rule for determining the contest score of the leader adjusts according to the number of net wins, and how important a win is (captured by the parameter $s$ ). This creates an additional asymmetry between the players that is not present in Konrad and Kovenock (2009); from Proposition 1 it is apparent that this factor reduces the expected efforts of both the leader and the laggard. The interplay between these two effects in our model allows for interesting predicted behavior depending upon the magnitude of the head start that is built up, and the number of contests that remain in the series.

We have focused on cases in which the laggard may be expected to exert more effort and find this to be most likely when he is at a large disadvantage (due to the leader relaxing), or when there are few contests remaining (since the value of remaining the leader diminishes). Due to the latter effect, the laggard will always be expected to exert more effort in the final contest. We can also show that, as long as the sequence is long enough (specifically, at least four contests), the laggard will be expected to have more effort already before the final contest. Should he subsequently lose in spite of this, the laggard will have more effort than the leader in the following contest.

We have been able to identify various patterns of expected effort. For example, the loser of an uneven contest will have more effort in the subsequent contest whether he is leader or laggard. Even a player who loses all contests will be expected to have larger effort than the rival at some stage 
before the final contest. These results are in contrast to the race literature in which a disadvantaged player will often simply give up.

We have considered several extensions to our main model to look at the robustness of our conclusions. Whereas our main model defines the win advantage as being in the form of a head start, we investigate an extension in which the advantage may be a handicap, or a combination of head start and handicap. The laggard can still have a higher effort than the leader in expectation, and this is more likely for a larger handicap, paralleling our previous result. The results of our main model are also robust to discounting. Finally, we show in an example that the restriction on having an identical prize in each contest can be relaxed, and that our results are robust as long as later prizes are not too small (in which case the laggard would again give up).

The latter is an interesting line of enquiry to pursue from a design point of view. A contest designer may be thought of as having a fixed budget to distribute in equal amounts over his chosen number of contests. In the course of the series of contests all rents are competed away in our symmetric model, so that the total amount of effort cannot be influenced. The timing of efforts can be influenced, however. The intensity of the initial fight depends upon the stage prize, the number of contests in the series, and the importance of each net win in determining the leader's score in the stage contest. To the extent that the importance of a win can be chosen by the designer, a larger value of this parameter will lead to a more intense fight since there is a larger value to becoming a leader. A larger stage prize and length of the contest series also leads to intense fighting initially. A contest designer may have a preference for ensuring that an early loser has the possibility of evening the score, so that the leader does not become very dominant. Here Corollary 7 can be used, setting the choice parameters so that a laggard gets an increased chance of winning the next contest.

Interesting design issues open up when we depart from our basic symmetric model, such as when the prize mass can be divided unequally between stage contests. Extending our model to account for heterogeneous contestants and asymmetric prizes in stage contests are topics addressed in our ongoing research; see Clark and Nilssen (2018).

\section{A Appendix}

\section{A.1 Proof of Proposition 2}

The payoff functions for contest $t$ are given in the text by (10) and (12). Using (5), we can stipulate the form of the equilibrium expected payoff for 
player 1 in contest $t$ to be:

$$
\begin{aligned}
u_{1, t}^{*}\left(M_{t}\right) & =u_{1, t+1}^{*}\left(M_{t}-1\right)+a_{t}+M_{t} s \\
& =u_{1, t+1}^{*}\left(M_{t}+1\right)+M_{t} s,
\end{aligned}
$$

where the second equality follows from (11). Using $u_{1, T}^{*}\left(M_{T}\right)=M_{T} s$ as calculated in the text, we can work out the equilibrium expected payoffs in each contest recursively backwards. This reveals the following pattern:

$$
\begin{aligned}
u_{1, T}^{*}\left(M_{T}\right) & =M_{T} s \\
u_{1, T-1}^{*}\left(M_{T-1}\right) & =\left(2 M_{T-1}+1\right) s \\
u_{1, T-2}^{*}\left(M_{T-2}\right) & =\left(3 M_{T-2}+3\right) s \\
u_{1, T-3}^{*}\left(M_{T-3}\right) & =\left(4 M_{T-3}+6\right) s
\end{aligned}
$$

For a specific contest $t$, this can be stated as

$$
u_{1, t}^{*}\left(M_{t}\right)=s\left[\sum_{j=0}^{T-t}\left(M_{t}+j\right)\right]=s\left[(T-t+1) M_{t}+\sum_{j=1}^{T-t} j\right]
$$

Working out the summation, this is rewritten in the more convenient form (19) in the Proposition.

In order to examine the equilibrium expected efforts for the advantaged and disadvantaged player, we simply need to identify the parameters in (4) for each contest. The bias term $z_{t}$ is $M_{t} s$, and we need to calculate the difference to the leader from winning and losing the current contest, $a_{t}$.

It is convenient to consider how $a_{t}$ is determined using (19). Recall (11):

$$
a_{t}=u_{1, t+1}^{*}\left(M_{t}+1\right)-u_{1, t+1}^{*}\left(M_{t}-1\right) .
$$

From (19), we have

$$
u_{1, t+1}^{*}\left(M_{t+1}\right)=s(T-t) M_{t+1}+\frac{1}{2}(T-t-1) .
$$

Applying (A3) in (A2), replacing $M_{t+1}$ by first $M_{t}+1$ and then $M_{t}-1$, gives

$$
\begin{aligned}
a_{t} & =s(T-t)\left[\left(M_{t}+1\right)-\left(M_{t}-1\right)\right] \\
& =2 s(T-t) .
\end{aligned}
$$

Putting $z=M_{t} s$ and $a=a_{t}$ into (4) gives the expected efforts in the Proposition. Note that the expected payoff of the laggard is zero, i.e., $p_{2, t}^{*} v-x_{2, t}^{*}\left(M_{t}\right)=0$. Using (16) gives the laggard's probability of winning.

In order to verify (20), we have, from (19), that

$$
u_{1, t}^{*}(1)=s(T-t+1)\left(1+\frac{1}{2}(T-t)\right) .
$$


From (14), each player's expected effort at $M_{t}=0$ is

$$
\begin{aligned}
\frac{1}{2}\left[v+u_{i, t+1}^{*}(1)\right] & =\frac{1}{2}\left\{v+s[T-(t+1)+1]\left[1+\frac{1}{2}[T-(t+1)]\right]\right\} \\
& =\frac{1}{2}\left[v+\frac{1}{2} s(T-t)(T-t+1)\right]
\end{aligned}
$$

where the first equality follows by advancing $u_{1, t}^{*}(1)$ one contest to $t+1$; this proves (20).

\section{A.2 Proof of Corollary 4}

Part (i): With $M_{t}=0$, total expected effort in contest $t$ is, by equation $(20)$,

$$
X_{t}^{*}(0)=v+\frac{1}{2} s(T-t)(T-t+1)>v,
$$

where the inequality follows from $t<T$.

Part (ii): With $M_{t}=1$, total expected effort is found from equations (15) and (16):

$$
\begin{aligned}
X_{t}^{*}(1) & =\frac{(v-s)^{2}}{2 v}+\frac{v^{2}-s^{2}}{2[v+2(T-t-1) s]} \\
& =(v-s)\left[\frac{v^{2}+(v-s)(T-t-1) s}{v^{2}+2 v(T-t-1) s}\right]<v-s .
\end{aligned}
$$

Since $2 v>v-s$, the fraction within square brackets in the second expression is less than 1 , and the inequality follows.

\section{A.3 Proof of Proposition 3}

Part (i). Suppose that player 1 is the leader. The laggard has more expected effort if condition (24) is fulfilled. This is least likely to be satisfied for $M_{t}=1$, in which case the condition can be written as

$$
t>T-\frac{v}{v-s} .
$$

Clearly, $T-\frac{v}{v-s}<T-1$, since $\frac{v}{v-s}>1$.

Part (ii). The laggard having more expected effort means, from (24), that

$$
M_{t}[v+s(T-t)]-v(T-t)>0 .
$$

If the laggard loses, then $M_{t+1}=M_{t}+1$, and the left hand side of the inequality for contest $t+1$ can be written as

$$
\begin{aligned}
\left(M_{t}+1\right)[v+s(T-t-1)]-v(T-t-1) & = \\
\left\{M_{t}[v+s(T-t)]-v(T-t)\right\}+\left[2 v-M_{t} s\right]+s(T-t-1) & >0,
\end{aligned}
$$


where the inequality follows since the curly-bracketed term is positive by (A4), and the square-bracketed one is positive by (7).

Part (iii). In contest $t$, we have $M_{t}[v+s(T-t)]-v(T-t)<0$, since the leader has more expected effort in this period. By the leader losing we get $M_{t+1}=M_{t}-1$, and the left hand side of the inequality for period $t+1$ becomes

$$
\begin{aligned}
\left(M_{t}-1\right)[v+s(T-t-1)]-v(T-t-1) & = \\
\left\{M_{t}[v+s(T-t)]-v(T-t)\right\}-M_{t} s-s(T-t-1) & <0 .
\end{aligned}
$$

Part (iv). If the laggard has more expected effort in contest $t$, then

$$
T-t<\frac{v M_{t}}{v-M_{t} s},
$$

by (24). If the laggard wins this contest, then $M_{t+1}=M_{t}-1$, and the leader has more expected effort in contest $t+1$ if

$$
T-t-1>\frac{v\left(M_{t}-1\right)}{v-\left(M_{t}-1\right) s} .
$$

For the inequalities in (A5) and (A6) to be consistent, we must have

$$
\begin{aligned}
\frac{v\left(M_{t}-1\right)}{v-\left(M_{t}-1\right) s}+1 & <\frac{v M_{t}}{v-M_{t} s} \Longleftrightarrow \\
s \frac{v\left(M_{t}-1\right)+\left[v-\left(M_{t}-1\right) s\right] M_{t}}{\left[v-\left(M_{t}-1\right) s\right]\left(v-M_{t} s\right)} & >0,
\end{aligned}
$$

which is clearly true, by (7).

\section{References}

Berger, J. and D. Pope (2011), "Can Losing Lead to Winning?", Management Science 57, 817-827.

Bergerhoff, J. and A. Vosen (2015), "Can Being Behind Get You Ahead? Reference Dependence and Asymmetric Equilibria in an Unfair Tournament", Discussion Paper 03/2015, Bonn Graduate School of Economics.

Clark, D.J. and T. Nilssen (2013), "Learning by Doing in Contests", Public Choice 156, 329-343.

Clark, D.J., and T. Nilssen (2018), "Beating the Matthew Effect: Head Starts and Catching Up in a Dynamic All-Pay Auction", Memorandum 02/2018, Department of Economics, University of Oslo. 
Clark, D.J., T. Nilssen, and J.Y. Sand (2018), "Dynamic Win Effects in Sequential Contests", unpublished manuscript.

Clark, D.J. and C. Riis (1995), "Social Welfare and a Rent-Seeking Paradox", Memorandum 23/1995, Department of Economics, University of Oslo.

Eriksson, T., A. Poulsen, and M.C. Villeval (2009), "Feedback and Incentives: Experimental Evidence", Labour Economics 16, 679-688.

Farrell, S. and A.R. Hakstian (2001), "Improving Salesforce Performance: A Meta-Analytic Investigation of the Effectiveness and Utility of Personnel Selection Procedures and Training Interventions", Psychology and Marketing 18, 281-316.

Franke, J., W. Leininger, and C. Wasser (2018), "Optimal Favoritism in All-Pay Auctions and Lottery Contests", European Economic Review 104, 22-37.

Fu, Q., J. Lu, and Y. Pan (2015), "Team Contests with Multiple Pairwise Battles", American Economic Review 105, 2120-2140.

Gelder, A. (2014), "From Custer to Thermopylae: Last Stand Behavior in Multi-Stage Contests", Games and Economic Behavior 87, 442-466.

Harris, C. and J. Vickers (1987), "Racing with Uncertainty", Review of Economic Studies 54, 1-21.

Hirata, D. (2014), "A Model of a Two-Stage All-Pay Auction", Mathematical Social Sciences 68, 5-13.

Joffrion, J.L. and S.O. Parreiras (2013), "A Theory of Dynamic Contests: Deadlines and Effort Smoothing", unpublished manuscript, US Air Force Academy and University of North Carolina at Chapel Hill.

Kirkegaard, R. (2012), "Favoritism in Asymmetric Contests: Head Starts and Handicaps", Games and Economic Behavior 76, 226-248.

Konrad, K.A., (2002), "Investment in the Absence of Property Rights: The Role of Incumbency Advantages", European Economic Review 46, $1521-1537$.

Konrad, K.A. (2009), Strategy and Dynamics in Contests. Oxford University Press.

Konrad, K.A. (2018), "Budget and Effort Choice in Sequential Colonel Blotto Campaigns", CESifo Economic Studies, forthcoming. 
Konrad, K. A. and D. Kovenock (2009), "Multi-Battle Contests", Games and Economic Behavior 66, 256-274.

Konrad, K.A. and D Kovenock (2010), "Contests with Stochastic Abilities", Economic Inquiry 48, 89-103.

Kovenock, D. and B. Roberson (2009), "Is the 50-State Strategy Optimal?", Journal of Theoretical Politics 21, 213-236.

Krishnamoorthy, A., S. Misra, and A. Prasad (2005), "Scheduling Sales Force Training: Theory and Evidence", International Journal of Research in Marketing 22, 427-440.

Krumer, A. (2013), "Best-of-Two Contests with Psychological Effects", Theory and Decision 75, 85-100.

Li, S. and J. Yu (2012), "Contests with Endogenous Discrimination", Economics Letters 117, 834-836.

Megidish, R. and A. Sela (2014), "Sequential Contests with Synergy and Budget Constraints", Social Choice and Welfare 42, 215-243.

Mehlum, H. and K.O. Moene (2006), "Fighting against the Odds", Economics of Governance 7, 75-87.

Mehlum, H. and K.O. Moene (2016), "Unequal Power and the Dynamics of Rivalry", Memorandum 13/2016, Department of Economics, University of Oslo.

Meirowitz, A. (2008), "Electoral Contests, Incumbency Advantages, and Campaign Finance", Journal of Politics 70, 681-699.

Ofek, E. and M. Sarvary (2003), "R\&D, Marketing, and the Success of Next-Generation Products", Marketing Science 22, 355-270.

Segev, E. and A. Sela (2014), "Sequential All-Pay Auctions with Head Starts", Social Choice and Welfare 43, 893-923.

Siegel, R. (2014), "Asymmetric Contests with Head Starts and Nonmonotonic Costs", American Economic Journal: Microeconomics 6(3), 59-105.

Skiera, B. and S. Albers (1998), "COSTA: Contribution Optimizing Sales Territory Alignment", Marketing Science 17, 196-213.

Strumpf, K.S. (2002), "Strategic Competition in Sequential Election Contests", Public Choice 111, 377-397. 
Tong, K. and K. Leung (2002), "Tournament as a Motivational Strategy: Extension to Dynamic Situations with Uncertain Duration", Journal of Economic Psychology 23, 399-420. 